\title{
Reconstruction of atomic columns in silicon by electron diffractive imaging
}

\author{
S. Morishita*, J. Yamasaki**, K. Nakamura*, T. Kato***, and N. Tanaka** \\ * Department of Crystalline Materials Science, Nagoya University, Nagoya, 464-8603, Japan \\ ** EcoTopia Science Institute, Nagoya University, Nagoya, 464-8603, Japan \\ *** Nanostructures Research Laboratory, Japan Fine Ceramics Center, Nagoya, 456-8587, Japan
}

In diffractive imaging, localized structures are reconstructed only from diffraction intensity by the iterative phase retrieval algorithms [1]. Since the first experimental result in 1999 [2], the method has been applied to some materials including biological ones [3] by using coherent x-ray radiation. The notable merit of diffractive imaging using electron beams is that image contrast and spatial resolutions in the reconstructed images are not influenced by lens aberrations. In the reconstruction process, a constraint in real space is generally imposed in such a way that the amplitudes of reconstructed wave field become zero in areas where no material exists. This means that diffractive imaging can be applied only to samples isolated in empty space such as nanotubes and nanowires [4][5]. It might be one of the reasons that there are only a few reports in which atomic-resolution images are reconstructed from electron diffraction patterns.

To avoid the restriction, we propose a new method applicable to samples in arbitrary shapes by using the selected area diffraction (SAD) method. In the method, a region where electron beams are intercepted by a selected area aperture is used for the constraint in real space. It is known that a spherical aberration $\left(\mathrm{C}_{\mathrm{s}}\right)$ of an objective lens generally causes area selection errors in SAD. To remove the errors, we use a $\mathrm{C}_{\mathrm{s}}$-corrector for imaging system. It enables us to select a well-defined area a few nanometers in diameter in a sample. We refer to the technique as selected area nanodiffraction (SAND) [6]. In the present experiments, a selected area aperture with a hole of $240 \mathrm{~nm}$ in diameter is inserted in the conjugate plane of the objective lens. Figure 1(a) shows a TEM image of the aperture hole, which selects an area about $3 \mathrm{~nm}$ in diameter in the sample plane. The corresponding electron diffraction pattern (Fig. 1(b)) does not completely coincide with Fraunhofer diffraction from a circular aperture, i.e., the Airy pattern, because of a partially coherent illumination. It is known that a high degree of coherence is required for diffractive imaging [7]. Figure 1(c) shows an intensity profile along the line in Fig. 1(b). As the result of accurate analysis of the profile, we have confirmed that a spatial coherence length of the illumination is more than twice as large as the diameter of the aperture [8].

A [011] SAND pattern from a silicon crystal is shown in Fig. 2(a). In the pattern, 400 and $\overline{4} 00$ spots corresponding to the separation of the dummbells are recorded. Noise and background intensity are removed before iterative reconstruction. Figure 2(b) shows the amplitude map of one of the exit wave fields reconstructed from the pattern. The alignment of atomic columns in silicon viewed from [011] is successfully reconstructed in the hole. The dumbbell structure with a separation of $0.136 \mathrm{~nm}$ is resolved clearly [9]. The shape of the aperture is also reconstructed well because of the pseudo Airy pattern convoluted on each diffraction spot in Fig. 2(a). We have found that averaging wave fields reconstructed from different initial phases improves quality of the result, for example, reduction of residual fluctuations between atomic columns in Fig. 2(b). It is concluded that the combination of SAND and diffractive imaging is effective in reconstructing nanostructures with atomic resolution, even if the samples are not surrounded by empty space, such as localized 
structures embedded in thin film samples. This means that the present method has a unique potential to expand the versatility of diffractive imaging by electron beams [10].

\section{References}

[1] R.W. Gerchberg, et al., Optik 35 (1972) 237.

[2] J. Miao, et al., Nature 400 (1999) 342.

[3] Y. Nishino, et al., Phys. Rev. Lett. 102 (2009) 018101.

[4] J.M. Zuo, et al., Science 300 (2003) 1419.

[5] O. Kamimura, et al., Appl. Phys. Lett. 92 (2008) 024106.

[6] J. Yamasaki, et al., J. Electron Microsc. 54 (2005) 123.

[8] J.C.H. Spence, et al., Ultramicroscopy 101 (2004) 149.

[7] S. Morishita, et al., in preparation.

[9] S. Morishita, et al., Appl. Phys. Lett. 93 (2008) 183103.

[10] The present study was partly supported by MEXT KAKENHI (18029011).
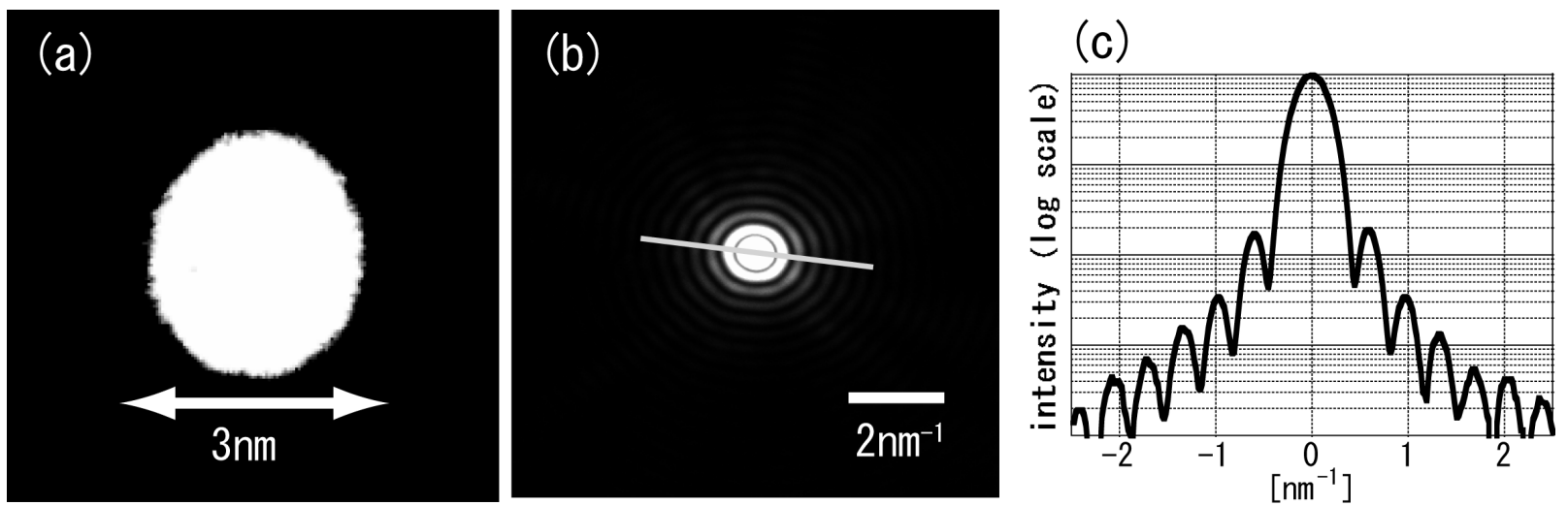

FIG. 1. (a) TEM image of the selected area aperture effectively about $3 \mathrm{~nm}$ in diameter. (b) Diffraction pattern from the aperture in (a). (c) Intensity profile along the line in (b).
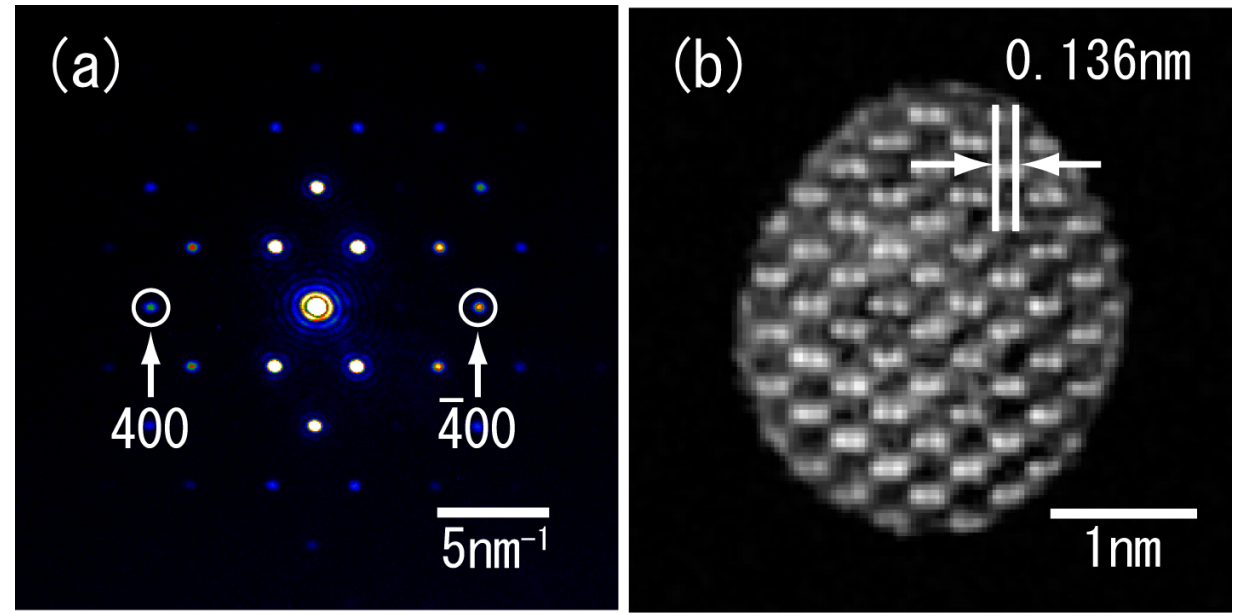

FIG. 2. (a) Selected area nano-diffraction pattern from a silicon crystal along [011]. (b) The amplitude map of a wave field reconstructed from (a). 